\title{
DISTRIBUCIÓN Y ESTADO DE CONSERVACIÓN DE LOS QUIRÓPTEROS EN ARAGÓN
}

\author{
J. T. Alcalde', D. Trujillo ${ }^{2}$ A. Artázcoz ${ }^{3} \&$ P. T. Agirre-Mendi ${ }^{4}$
}

\begin{abstract}
RESUMEN
En el período 2004-2006 se ha realizado un muestreo de los quirópteros de Aragón. Se ha trampeado en 47 bosques y se han inspeccionado 67 refugios potenciales. Para ello se han utilizado redes finas, trampas de arpa, detectores de ultrasonidos, focos y cámaras de grabación de vídeo.

Se han capturado 1197 ejemplares pertenecientes a 24 especies. Además se han obtenido 529 citas y se ha identificado la presencia de al menos 120 colonias reproductoras (32 de ellas han sido localizadas). Las especies más frecuentes son fisurícolas y ubiquistas: el murciélago enano (Pipistrellus pipistrellus, 110 citas y 30 colonias de cría), el montañero (Hypsugo savii, 63 citas y 11 agrupaciones) y el de borde claro (Pipistrellus kuhlii, 48 citas y 13 colonias). Se han encontrado datos de reproducción para todas las especies identificadas salvo Myotis capaccinii, Myotis cf. nattereri, Nyctalus lasiopterus, Nyctalus leisleri y Eptesicus serotinus.

Las especies halladas se reparten según cuatro patrones: uno de distribución general y continua ( $P$. pipistrellus, $P$. kuhlii, H. savii, E. serotinus y $P$. austriacus), otro de distribución general pero discontinua ( $R$. ferrumequinum, $R$. hipposideros, $R$. euryale, $M$. myotis, M. blythii, M. escalerae, M. emarginatus, M. daubentonii, P. pygmaeus, M. schreibersii y T. teniotis), otro de especies forestales, presentes únicamente en algunas de las grandes manchas boscosas (Pirineos, Moncayo y sur de Teruel: $M$. mystacinus, $M$. cf. nattereri, $P$. auritus, B. barbastellus, $N$. lasiopterus y $N$. leisleri) y el último con dos especies de presencia muy restringida en la región ( $M$. capaccinii y $P$. macrobullaris).

Se muestra la distribución de estas especies en Aragón y se comenta su estado de conservación en función de los datos obtenidos en este trabajo y la bibliografía.
\end{abstract}

Palabras clave: quirópteros, Aragón, distribución, conservación.

\section{ABSTRACT \\ Distribution and status of Chiroptera in Aragón}

In the period 2004-2006 a sampling of bats took place in Aragón. Traps were set in 47 forests and 67 potential shelters were inspected. Mist nets, harp traps, ultrasound detectors and video cameras were used.

A total of 1197 specimens, belonging to 24 species, were captured; 529 records were obtained and the presence of at least 120 breeding colonies was identified (32 of them

Plaza Guitarrista Sabicas, 5, 2º B - 31015 Pamplona (Navarra) jtalcalde@wanadoo.es

C/José Betancor Cabrera, 20 - 38410 Los Realejos (Tenerife)

C/Navarro Villoslada, 11, $5^{\circ} \mathrm{D}-31003$ Pamplona (Navarra)

C/ Paseo del Río Grande, 7, $3^{\circ}$ I - 01320 Oyón-Oion (Álava). 
were found). The most frequent species are those that roost in crevices and are ubiquists: the common pipistrelle (Pipistrellus pipistrellus, 110 records and 30 breeding colonies), Savi's pipistrelle (Hypsugo savii, 63 records and 11 colonies) and Kuhl's pipistrelle (Pipistrellus kuhlii, 48 records and 13 colonies). Reproduction data have been found for all species except for Myotis capaccinii, Myotis cf. nattereri, Nyctalus lasiopterus, Nyctalus leisleri and Eptesicus serotinus.

The species found can be divided into four large groups: one of general and continuous distribution ( $P$. pipistrellus, $P$. kuhlii, H. savii, E. serotinus and $P$. austriacus), another of general but discontinuous distribution ( $R$. ferrumequinum, $R$. hipposideros, $R$. euryale, M. myotis, M. blythii, M. escalerae, M. emarginatus, $M$. daubentonii, $P$. pygmaeus, M. schreibersii and T. teniotis), a third of forest species, which were found only in some of the extensive wooded areas (Pyrenees, Moncayo and the south of Teruel: $M$. mystacinus, $M$. cf. nattereri, P. auritus, B. barbastellus, $N$. lasiopterus and $N$. leisleri) and finally two very rare species in the region (M. capaccinii and P. macrobullaris).

The distribution of these species in Aragon is shown and their status in relation to data obtained and the bibliography is reviewed.

Key words: Chiroptera, Aragon, distribution, conservation.

\section{Introducción}

A pesar de su gran extensión (más de 47.500 $\mathrm{km}^{2}$ ), la gran diversidad de hábitats y la abundancia de refugios apropiados, Aragón es un territorio escasamente estudiado todavía en relación a los quirópteros. Los primeros trabajos (Navas, 1901; Cabrera, 1914; Maynar, 1919; Balcells, 1965) aportan pocos datos para la región y se centran principalmente en murciélagos cavernícolas. En las dos últimas décadas se han publicado diversos estudios que han añadido nuevas citas, aunque estas prospecciones no abarcan todo el territorio (Ibáñez \& Fernández, 1989; Trujillo, 1991; Serra-Cobo et al., 1992; Bafaluy Zoriguel, 1997, 1999, 2000; Bafaluy Zoriguel et al., 1997). Recientemente destacan el trabajo de Woutersen \& Bafaluy Zoriguel (2001), que reúne numerosos datos de 23 especies presentes en Huesca, y el de Garin et al. (2003), que menciona una nueva especie para la península Ibérica, descubierta en el Pirineo aragonés.

El presente trabajo tiene como objetivo mejorar el conocimiento de los murciélagos de toda la región, aportando nuevos datos sobre su distribución, biología y estado de conservación.

\section{Material y métodos}

En los veranos del período 2004-2006 se han realizado muestreos nocturnos de los murciélagos presentes en diferentes puntos de 47 bosques repartidos por todo Aragón. Dado que cada método de identificación es selectivo para determinadas especies (Nature Conservation Council, 1987; Kunz \&
Kurta, 1988), se han empleado metodologías diversas: detectores de ultrasonidos con sistemas de heterodinaje y tiempo expandido (D240x, D960 y D1000x, Pettersson Elektronik AB) conectados a grabadoras o cámaras de vídeo; redes finas sobre ríos, estanques y charcas, en el interior del bosque (generalmente atravesando pistas forestales) o a la entrada de refugios; trampas de arpa colocadas sobre el agua o en la boca de acceso a refugios; reclamos de ultrasonidos colocados junto a redes o arpas. Además en verano e invierno se han inspeccionado 67 refugios potenciales con focos y detectores de ultrasonidos. En total, se ha muestreado en 170 puntos situados entre 73 y 1966 m.s.n.m. (media $=815 \mathrm{~m} ; \mathrm{s}=445 \mathrm{~m}$.) Ver Figura 1 .

La identificación de los ejemplares se ha realizado por tres medios: en mano, tomando biometrías y otros rasgos característicos de los individuos capturados, mediante observación directa en los refugios y realizando análisis de ultrasonidos de especies fácilmente identificables (géneros Rhinolophus, Pipistrellus, Hypsugo, Nyctalus, Eptesicus, Barbastella y Tadarida). En total se han grabado y analizado más de 500 series de ultrasonidos. Para ello se ha empleado un programa informático específico (Bat Sound 3.31, Pettersson Elektronik AB) y se ha medido la frecuencia de máxima energía de los pulsos, su duración y ritmo (Ahlén, 1990; Barataud, 1996; Russo \& Jones, 2002).

Teniendo en cuenta la reciente mención de nuevas especies crípticas de quirópteros ibéricos (Ibáñez et al., 2006), la identificación de Pipistrellus pygmaeus, Myotis mystacinus, Myotis escalerae, Myotis cf. nattereri y Plecotus macrobullaris, se ha confirmado mediante análisis de ADN mito- 


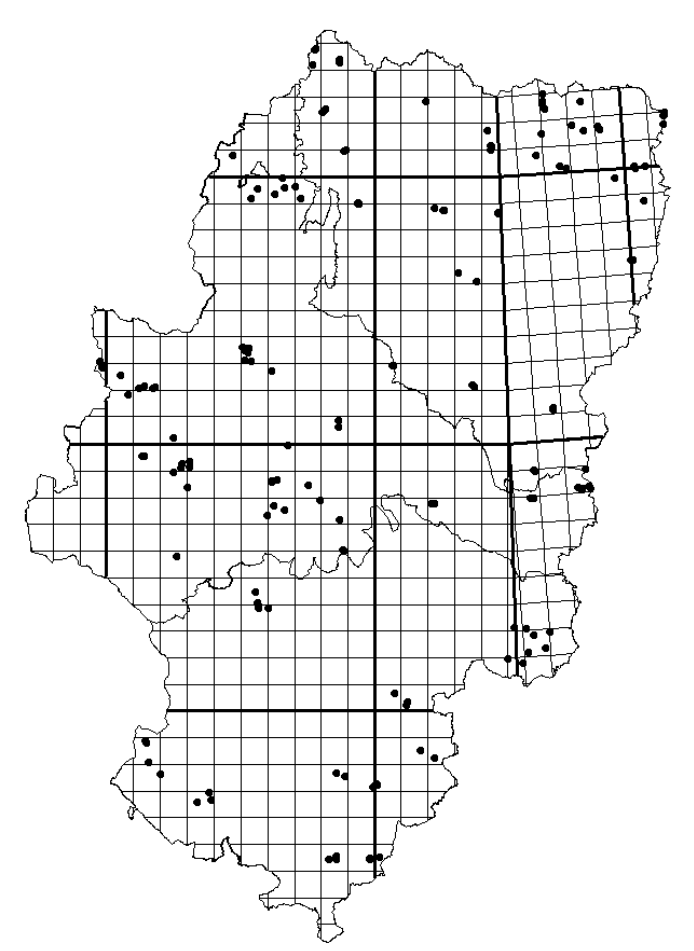

Fig. 1.- Puntos de muestreo en el presente trabajo.

Fig. 1.- Sampling places used in this work.

condrial llevados a cabo por investigadores de la Estación Biológica de Doñana (CSIC). También se han realizado análisis similares para confirmar especies, subespecies o linajes de Pipistrellus kuhlii, Hypsugo savii, Plecotus auritus, Plecotus austriacus y Eptesicus serotinus.

Los animales capturados han sido examinados y se ha determinado el sexo, la edad (joven del año o adulto) y el estado reproductor, siendo liberados posteriormente en el lugar de captura. Se ha asumido que la captura de uno o varios individuos reproductores o sus crías en una zona supone la existencia de al menos una colonia reproductora. También se han identificado colonias en sus refugios. El número y tamaño de estas agrupaciones resulta básico para poder discernir el estado de las poblaciones de cada especie. Las colonias cavernícolas halladas, han sido censadas mediante grabaciones de vídeo con luz infrarroja y detector de ultrasonidos.

La distribución de cada especie se muestra en mapas de cuadrícula UTM de $10 \mathrm{~km}$ de lado; en ellos se han incluido todas las citas bibliográficas

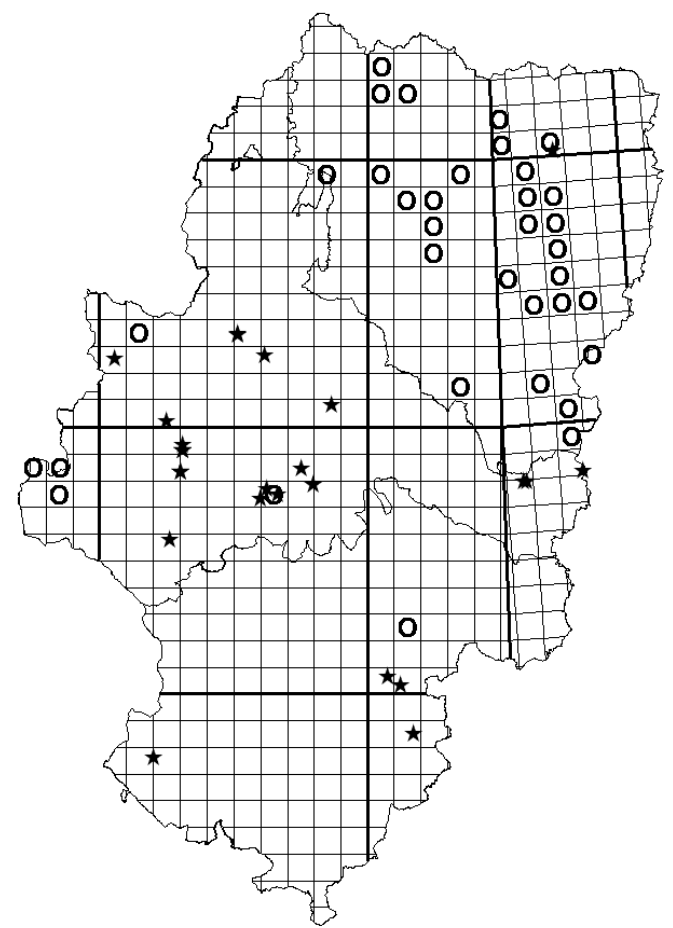

Fig. 2.- Observaciones de Rhinolophus ferrumequinum. Los círculos blancos indican citas bibliográficas y las estrellas los datos aportados en este trabajo.

Fig. 2.- Records of Rhinolophus ferrumequinum. Open circles show literature records and stars show records in this work.

(con círculos centrados en cada cuadrícula) a excepción de algunas observaciones de Woutersen \& Bafaluy Zoriguel (2001) que se consideran dudosas (determinaciones visuales de algunas especies que requieren su identificación en mano: Myotis mystacinus, Myotis bechsteinii, Plecotus auritus y Plecotus austriacus). Los datos del presente trabajo se indican con estrellas en la situación exacta.

\section{Resultados}

Se han capturado 1197 ejemplares (702 machos y 495 hembras) y se han obtenido 529 citas (especielocalidad) pertenecientes a 24 especies de quirópteros (Tabla 1). Además se ha identificado la presencia de al menos 120 colonias reproductoras, de las que 32 han sido localizadas en sus refugios (Tabla 2). Se menciona por vez primera la presencia de Myotis escalerae y Myotis cf. nattereri en Aragón. 
Tabla 1.- Número de citas obtenidas con cada método. "En refugio" indica las capturas dentro de un abrigo. *: detector y observación.

Table 1.- Number of records obtained with each method. "En refugio" indicates captures inside a shelter. *: detector and observation.

\begin{tabular}{|c|c|c|c|c|c|c|}
\hline Especie & Arpa & Detector & Observación & Red & En refugio & TOTAL \\
\hline Rhinolophus ferrumequinum (Schreber, 1774) & 4 & 0 & 18 & 1 & 0 & 23 \\
\hline Rhinolophus hipposideros (Bechstein, 1800) & 1 & 6 & 10 & 1 & 0 & 18 \\
\hline Rhinolophus euryale (Blasius, 1853) & 1 & 0 & 11 & 0 & 0 & 12 \\
\hline Myotis myotis Borkhausen, 1797 & 2 & 0 & 0 & 10 & 5 & 17 \\
\hline Myotis blythii (Tomes, 1857) & 0 & 0 & 0 & 1 & 2 & 3 \\
\hline Myotis escalerae Cabrera, 1904 & 3 & 0 & 0 & 5 & 5 & 13 \\
\hline Myotis cf. nattereri & 0 & 0 & 0 & 1 & 0 & 1 \\
\hline Myotis cf. nattereri/escalerae & 2 & 0 & 0 & 7 & 0 & 9 \\
\hline Myotis emarginatus (Geoffroy, 1806) & 1 & 0 & 3 & 5 & 0 & 9 \\
\hline Myotis mystacinus (Kuhl, 1817) & 3 & 0 & 0 & 3 & 0 & 6 \\
\hline Myotis daubentonii (Kuhl, 1817) & 2 & 0 & 2 & 12 & 0 & 16 \\
\hline Myotis capaccinii (Bonaparte, 1837) & 0 & 0 & 0 & 2 & 0 & 2 \\
\hline Pipistrellus pipistrellus (Schreber, 1774) & 12 & 47 & 0 & 49 & 2 & 110 \\
\hline Pipistrellus pygmaeus (Leach, 1825) & 2 & $7 *$ & 0 & 4 & 0 & 13 \\
\hline Pipistrellus kuhlii (Kuhl, 1819) & 1 & 28 & 0 & 19 & 0 & 48 \\
\hline Hypsugo savii (Bonaparte, 1837) & 7 & 19 & 2 & 35 & 0 & 63 \\
\hline Nyctalus leisleri (Kuhl, 1817) & 0 & 5 & 0 & 11 & 0 & 16 \\
\hline Nyctalus lasiopterus (Schreber, 1780) & 0 & $1 *$ & 0 & 6 & 0 & 7 \\
\hline Eptesicus serotinus (Schreber, 1774) & 1 & 15 & 0 & 13 & 0 & 29 \\
\hline Barbastella barbastellus (Schreber, 1774) & 5 & 4 & 2 & 5 & 0 & 16 \\
\hline Plecotus auritus (Linnaeus, 1758) & 5 & 0 & 0 & 13 & 1 & 19 \\
\hline Plecotus austriacus (Fischer, 1829) & 4 & 0 & 3 & 22 & 0 & 29 \\
\hline Plecotus macrobullaris Kuzjakin, 1965 & 0 & 0 & 0 & 1 & 0 & 1 \\
\hline Miniopterus schreibersii (Kuhl, 1817) & 1 & 0 & 3 & 5 & 0 & 9 \\
\hline Tadarida teniotis (Rafinesque, 1814) & 0 & 38 & 0 & 2 & 0 & 40 \\
\hline TOTAL & 57 & 170 & 54 & 233 & 15 & 529 \\
\hline
\end{tabular}

A continuación se exponen los datos obtenidos para cada especie, agrupándolas por el tipo de refugio que utilizan. En cada grupo, las especies se nombran por orden sistemático.

\section{ESPECIES CAVERNÍCOLAS}

El murciélago grande de herradura, Rhinolophus ferrumequinum ( 23 citas, 4 colonias de cría en edificios, Fig. 2), es frecuente aunque se halla de forma discontinua, en función de la disponibilidad de refugios adecuados (abrigos subterráneos y construcciones deshabitadas principalmente). Las colonias halladas oscilan entre 14 y 350 ejemplares adultos.

El murciélago pequeño de herradura, Rhinolophus hipposideros (18 citas, 5 colonias reproductoras en edificios, Fig. 3), presenta una distribución también general pero discontinua. Las colonias encontradas son pequeñas (entre 7 y 30 individuos).
El murciélago mediterráneo de herradura, Rhinolophus euryale (12 citas, 3 colonias reproductoras en edificios, Fig. 4), resulta menos frecuente que las especies anteriores y se distribuye de forma más dispersa, por todo el área de estudio. Las colonias contienen de 20 a 60 ejemplares.

El ratonero grande, Myotis myotis (17 citas, 1 colonia en una cueva, Fig. 5), se encuentra muy disperso; la única colonia reproductora hallada contenía alrededor de 1000 individuos adultos.

El murciélago ratonero mediano, Myotis blythii ( 3 citas, 1 colonia reproductora identificada pero no localizada, Fig. 6), parece escaso e infrecuente en toda la región, excepto en el sur de Huesca, donde se han hallado 4 colonias de considerable tamaño (Woutersen \& Bafaluy Zoriguel, 2001). Su distribución es muy dispersa.

La situación de la especie considerada como Myotis nattereri en Iberia es todavía incierta. Ibáñez 
3

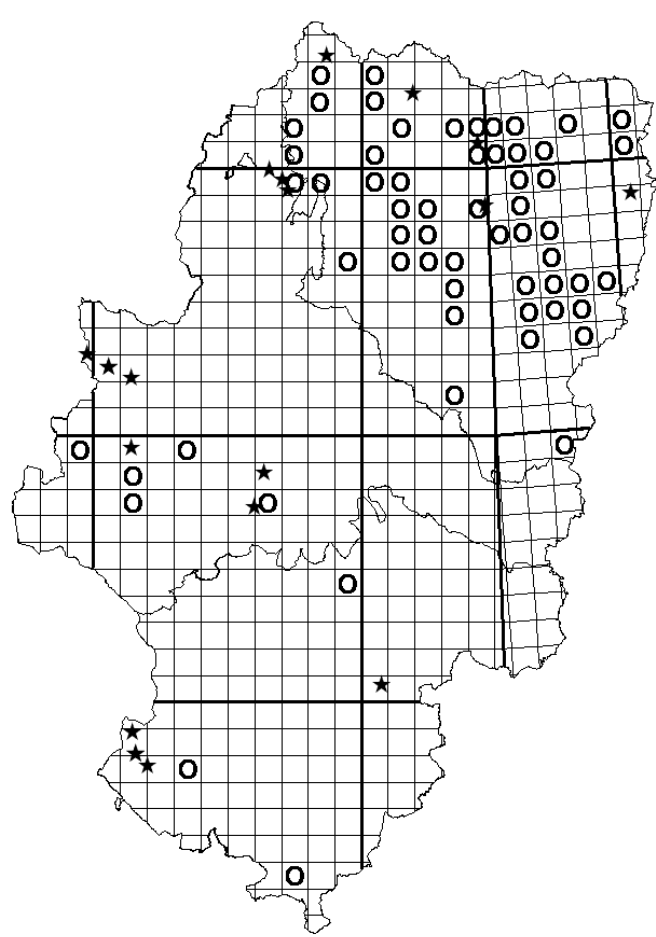

5

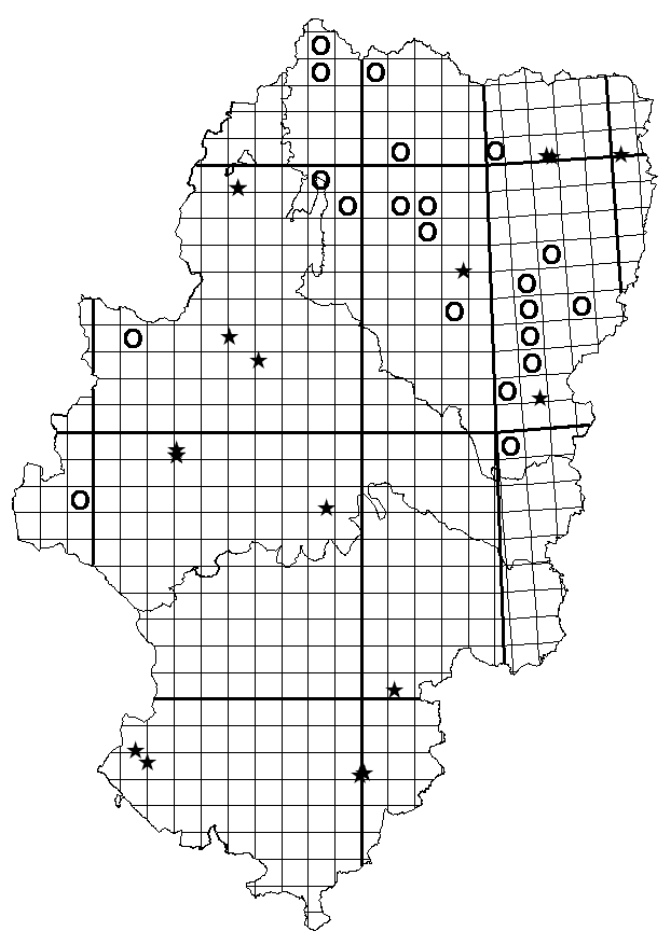

4

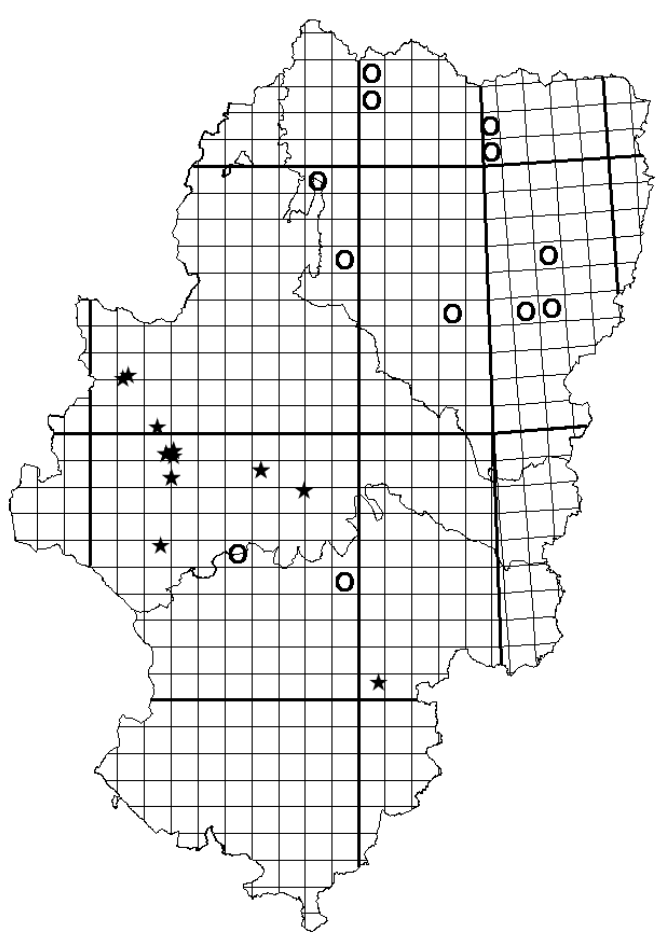

6

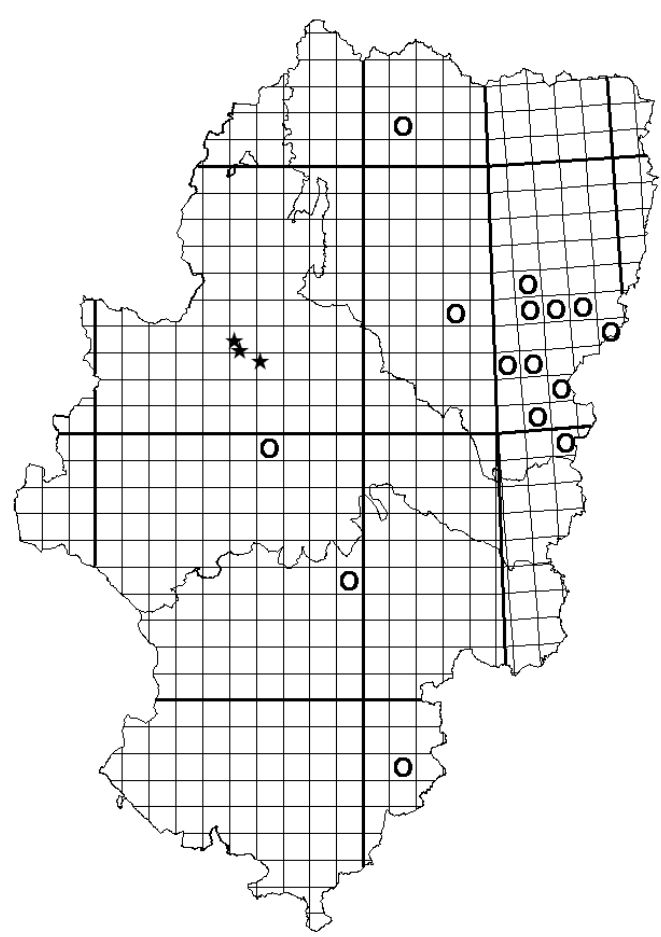

Fig. 3-6.- Observaciones de Rhinolophus hipposideros (3), R. euryale (4), Myotis myotis (5) y M. blythii (6). Símbolos como en la Fig. 2.

Fig. 3-6.- Records of Rhinolophus hipposideros (3), R. euryale (4), Myotis myotis (5) and M. blythii (6). Symbols as in Figure 2. 
Tabla 2.- Principales datos de las observaciones realizadas en el presente trabajo. Se indica el número mínimo de colonias reproductoras identificadas obtenido por captura de hembras criando y entre paréntesis el número de colonias reproductoras localizadas en sus refugios.

Table 2.- Main data of records obtained in this work. The minimum number of identified reproductive colonies obtained by the capture of breeding females is shown, and in brackets, the number of breeding colonies found in their shelters.

\begin{tabular}{|c|c|c|c|c|c|c|}
\hline Especies & $\begin{array}{c}\mathbf{N} \\
\text { capturas }\end{array}$ & $\begin{array}{l}\text { Citas } \\
\text { trabajo }\end{array}$ & $\begin{array}{c}\mathrm{N} \text { colonias } \\
\text { reproductoras }\end{array}$ & $\begin{array}{l}\% \text { citas } \\
\text { trabajo }\end{array}$ & $\begin{array}{c}\text { Rango } \\
\text { altitudinal }\end{array}$ & $\begin{array}{c}\text { Altitud } \\
\text { media }\end{array}$ \\
\hline Rhinolophus ferrumequinum & 36 & 23 & $4(4)$ & 4,3 & $149-1597$ & 610 \\
\hline Rhinolophus hipposideros & 16 & 18 & $5(5)$ & 3,4 & $556-1599$ & 980 \\
\hline Rhinolophus euryale & 3 & 12 & $4(4)$ & 2,3 & $509-792$ & 653 \\
\hline Myotis myotis & 30 & 17 & $1(1)$ & 3,2 & $160-1965$ & 870 \\
\hline Myotis blythii & 12 & 3 & $1(0)$ & 0,6 & $224-269$ & 251 \\
\hline Myotis escalerae & 20 & 13 & $7(4)$ & 2,5 & $534-1597$ & 855 \\
\hline Myotis cf. nattereri & 2 & 1 & 0 & 0,2 & 1010 & 1010 \\
\hline Myotis cf. nattereri/escalerae & 12 & 9 & $4(2)$ & 1,7 & $868-1965$ & 1373 \\
\hline Myotis emarginatus & 10 & 9 & $5(2)$ & 1,7 & $123-1599$ & 716 \\
\hline Myotis mystacinus & 11 & 6 & $1(0)$ & 1,1 & $656-1965$ & 1417 \\
\hline Myotis daubentonii & 57 & 16 & $4(0)$ & 3,0 & $367-1240$ & 852 \\
\hline Myotis capaccinii & 2 & 2 & 0 & 0,4 & $150-367$ & 259 \\
\hline Pipistrellus pipistrellus & 321 & 110 & $30(2)$ & 20,8 & $122-1966$ & 934 \\
\hline Pipistrellus pygmaeus & 23 & 13 & $4(3)$ & 2,5 & $132-782$ & 298 \\
\hline Pipistrellus kuhlii & 60 & 48 & $13(2)$ & 9,1 & $122-1966$ & 818 \\
\hline Hypsugo savii & 277 & 63 & $11(0)$ & 11,9 & $224-1966$ & 1030 \\
\hline Nyctalus leisleri & 49 & 16 & 0 & 3,0 & $868-1819$ & 1191 \\
\hline Nyctalus lasiopterus & 16 & 7 & 0 & 1,3 & $660-1483$ & 1093 \\
\hline Eptesicus serotinus & 28 & 29 & 0 & 5,5 & $122-1500$ & 759 \\
\hline Barbastella barbastellus & 26 & 16 & $4(1)$ & 3,0 & $160-1521$ & 1086 \\
\hline Plecotus auritus & 68 & 19 & $9(0)$ & 3,6 & $1010-1965$ & 1372 \\
\hline Plecotus austriacus & 77 & 29 & $9(0)$ & 5,5 & $160-1483$ & 963 \\
\hline Plecotus macrobullaris & 11 & 1 & $1(0)$ & 0,2 & 1819 & 1819 \\
\hline Miniopterus schreibersii & 19 & 9 & $2(2)$ & 1,7 & $534-1599$ & 982 \\
\hline Tadarida teniotis & 11 & 40 & $1(0)$ & 7,6 & 224-1599 & 901 \\
\hline$\overline{T O T A L}$ & 1197 & 529 & $120(32)$ & 100,0 & $122-1966$ & 815 \\
\hline
\end{tabular}

et al. (2006) reconocen la presencia de al menos dos linajes ibéricos con categoría de especie, Myotis escalerae y Myotis cf. nattereri (Fig. 7). En el presente trabajo se han encontrado 13 observaciones ( 7 colonias identificadas, 3 localizadas en cuevas y una más en un puente) de Myotis escalerae, que se reparte de forma general y dispersa por toda la región; las colonias oscilan entre 50 y 880 ejemplares adultos. Por el contrario, sólo se ha realizado una observación (2 machos) de Myotis cf. nattereri, en Pirineos. Además se han obtenido datos de Myotis escalerae / cf. nattereri en otras 9 localidades (4 colonias identificadas, 2 localizadas en cuevas) aunque no se ha podido determinar la especie.

El murciélago ratonero pardo, Myotis emarginatus (9 citas, 5 colonias identificadas, Fig. 8), se encuentra de forma dispersa por toda la región. Las dos únicas colonias localizadas contienen entre 25 y 100 ejemplares adultos.

El murciélago ratonero patudo, Myotis capaccinii (2 citas, Fig. 9), es muy escaso y de distribución restringida: se halla en el sur de Huesca y este de Zaragoza, lo que constituye el límite noroccidental de su área de presencia en España (Almenar et al., 2002). Aunque en este trabajo no se han identificado colonias reproductoras, existen datos de su cría en Huesca (Woutersen \& Bafaluy Zoriguel, 2001).

El murciélago de cueva, Miniopterus schreibersii (9 citas, 2 colonias en cuevas, Fig. 10), ciñe su distribución a la existencia de cavidades adecuadas. No obstante, su veloz vuelo le permite alejarse rápidamente de sus refugios, por lo que también se ha encontrado cazando en zonas relativamente lejanas. Es muy gregario y su población se concentra en 
7

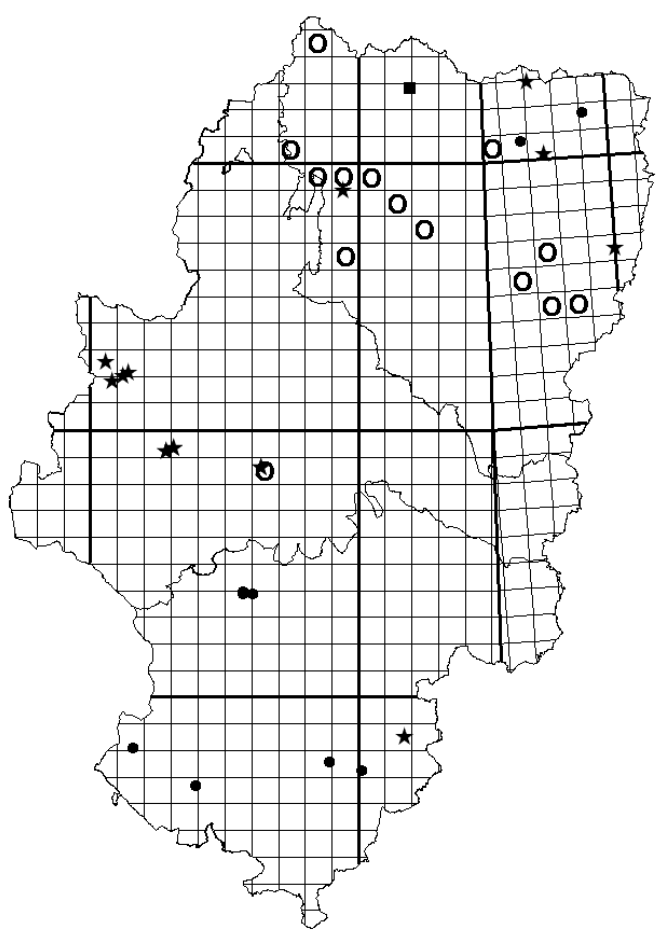

9

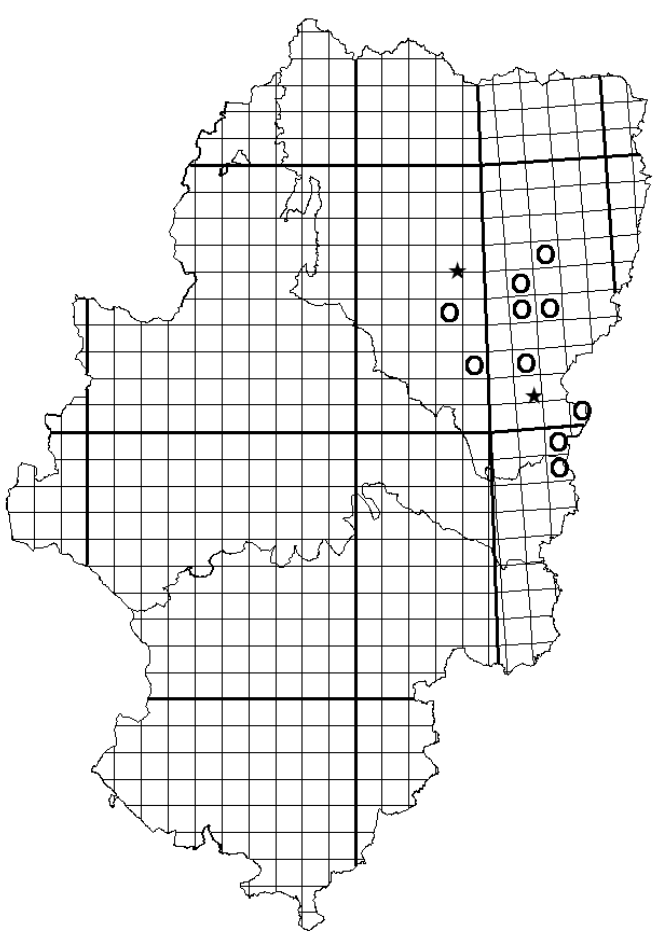

8

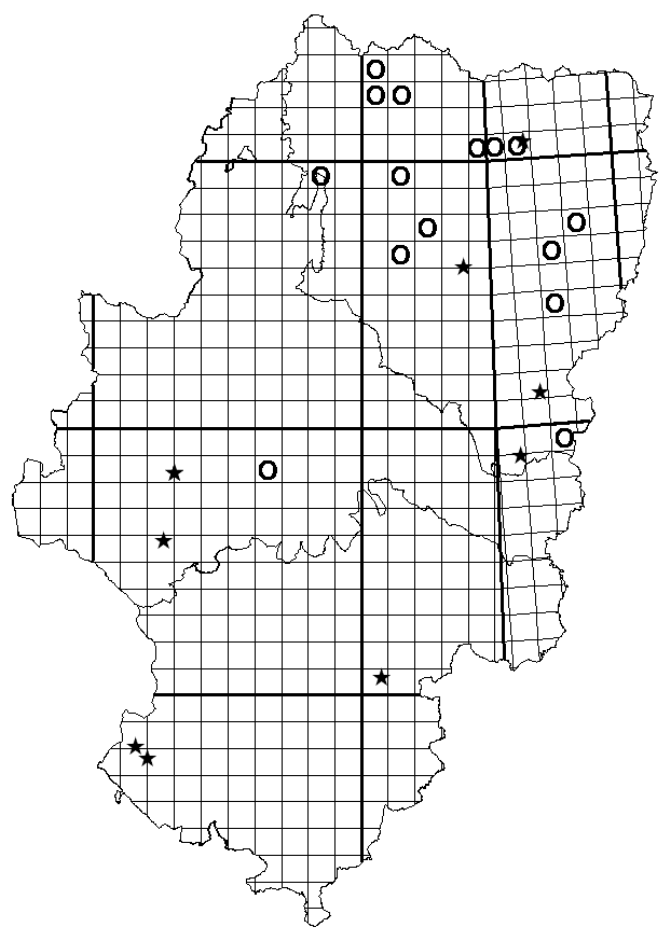

10

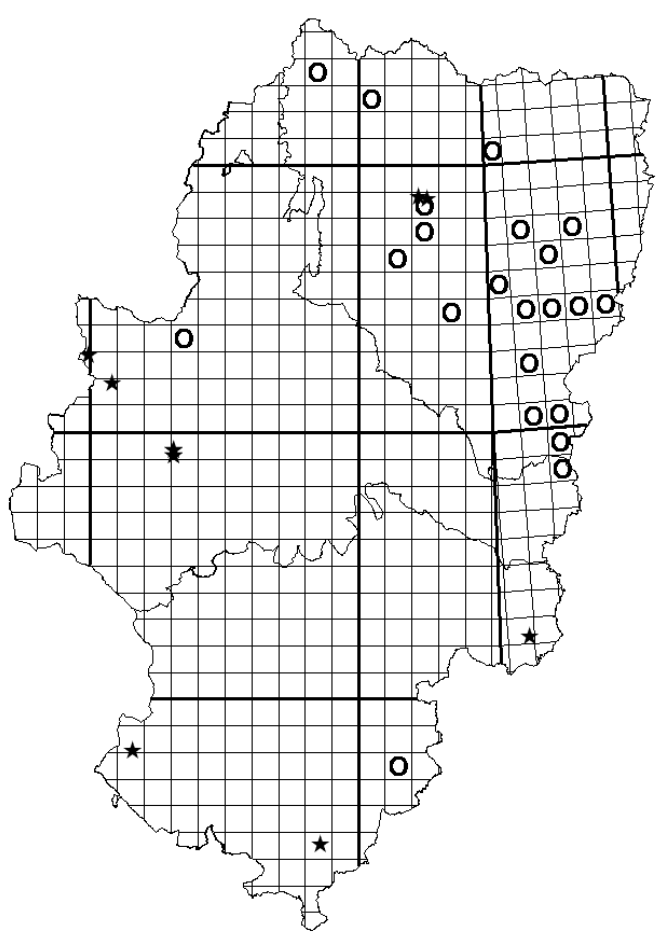

Fig. 7-10.- Observaciones de Myotis escalerae (estrellas) y M. cf. nattereri (cuadrados) (7) (los círculos negros indican citas de cualquiera de las dos especies; los círculos blancos, datos bibliográficos), M. emarginatus (8), M. capaccinii (9) y Miniopterus schreibersii (10). Símbolos como en la Fig. 2.

Fig. 7-10.- Records of Myotis escalerae (stars) and M. cf. nattereri (squares) (7) (closed circles show records of either of these species; open circles show data from literature), M. emarginatus (8), M. capaccinii (9) and Miniopterus schreibersii (10). Symbols as in Fig. 2. 
11

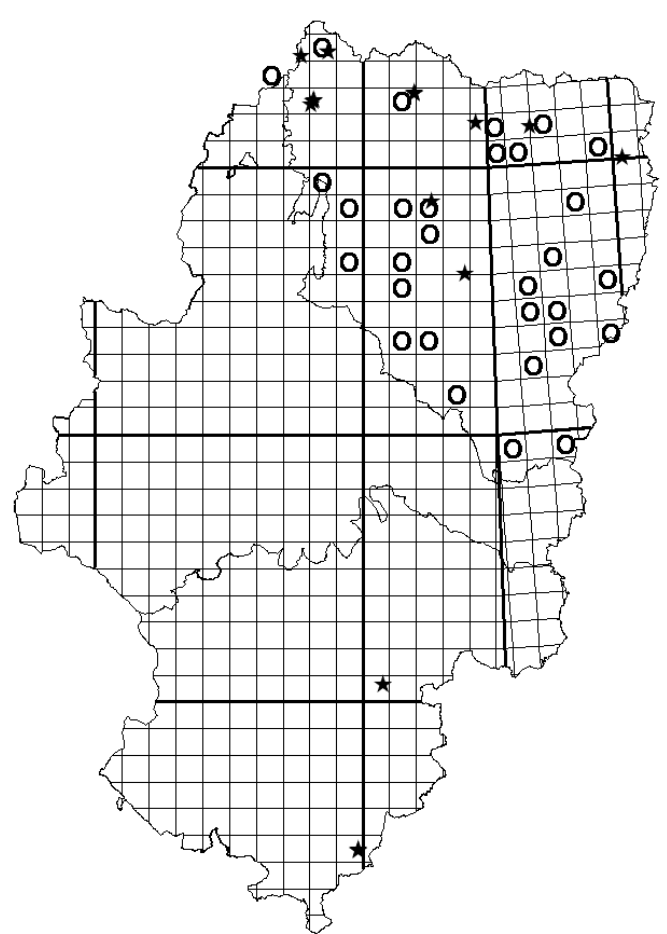

12

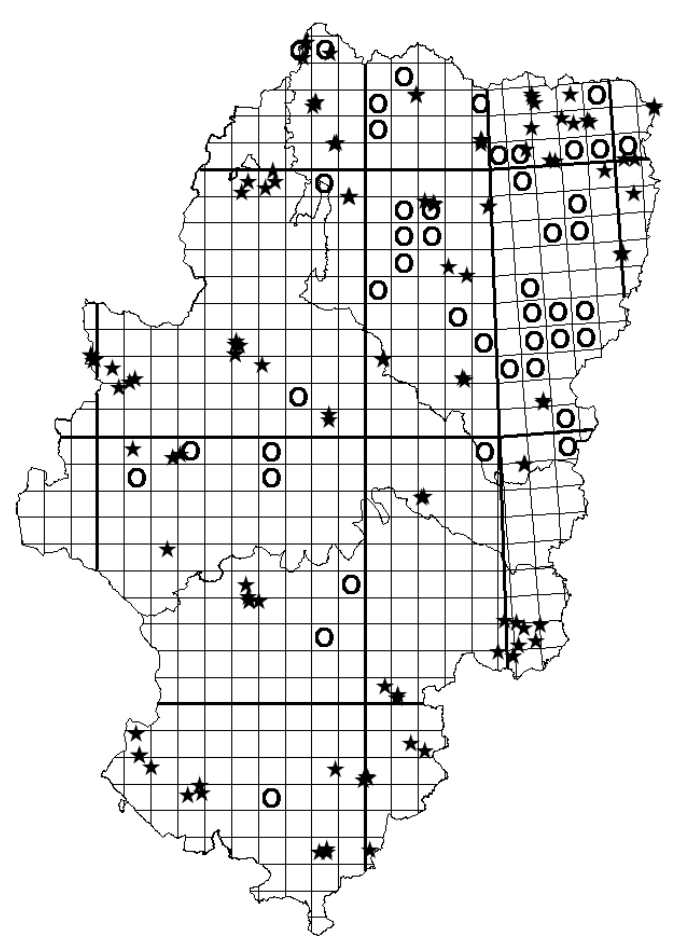

Fig. 11-12._- Observaciones de Myotis daubentonii (11) y Pipistrellus pipistrellus (12). Símbolos como en la Fig. 2.

Fig. 11-12.- Records of Myotis daubentonii (11) and Pipistrellus pipistrellus (12). Symbols as in Fig. 2.

muy pocas colonias reproductoras, muy numerosas: las dos colonias censadas contenían 600 y 4000 individuos.

\section{ESPECIES FISURÍCOLAS}

El murciélago ratonero ribereño, Myotis daubentonii (16 citas, 4 colonias identificadas, Fig. 11), caza principalmente en cursos y masas de agua de tamaño medio o pequeño (Boyero, 2002), por lo que su presencia se restringe a estas zonas, donde es frecuente (Pirineo, Prepirineo y sureste de Teruel). No se ha hallado en el Ebro, aunque Woutersen \& Bafaluy Zoriguel (2001) lo mencionan en sus cercanías.

El murciélago enano, Pipistrellus pipistrellus (110 citas, 30 colonias reproductoras, Fig. 12), es ubiquista y se distribuye de forma amplia y continua por toda la región.

El murciélago de Cabrera, Pipistrellus pygmaeus (13 citas, 4 colonias, Fig. 13), se ha localizado en las riberas de ríos de baja altitud (especialmente en el valle del Ebro), donde resulta muy abundante. Falta en los demás hábitats, análogamente a como lo encontramos en La Rioja (Agirre-Mendi \& Ibáñez, 2004). Se han identificado 2 colonias reproductoras en sendos huecos de álamos y una gran colonia de aproximadamente 3000 ejemplares en las grietas de un puente sobre el Ebro.

El murciélago de borde claro, Pipistrellus kuhlii, es frecuente (48 citas, 13 colonias, Fig. 14) y se encuentra de forma continua en todo Aragón. Destaca una observación realizada a 1966 m.s.n.m., por ser la máxima altitud registrada para la especie en la península Ibérica hasta la fecha (Goiti \& Garin, 2002). Todos los P. kuhlii identificados pertenecen al linaje ibérico detectado molecularmente por Ibáñez et al. (2006).

El murciélago montañero, Hypsugo savii (63 citas, 11 colonias, Fig. 15), resulta también frecuente, especialmente en los cañones y roquedos, hallándose de forma prácticamente continua por todo el territorio. Todos los $H$. savii identificados pertenecen al linaje ibérico detectado molecularmente por Ibáñez et al. (2006).

El murciélago hortelano, Eptesicus serotinus, es también frecuente (29 citas, Fig. 16) y ubiquista. Aunque no se ha identificado la presencia de colo- 
13

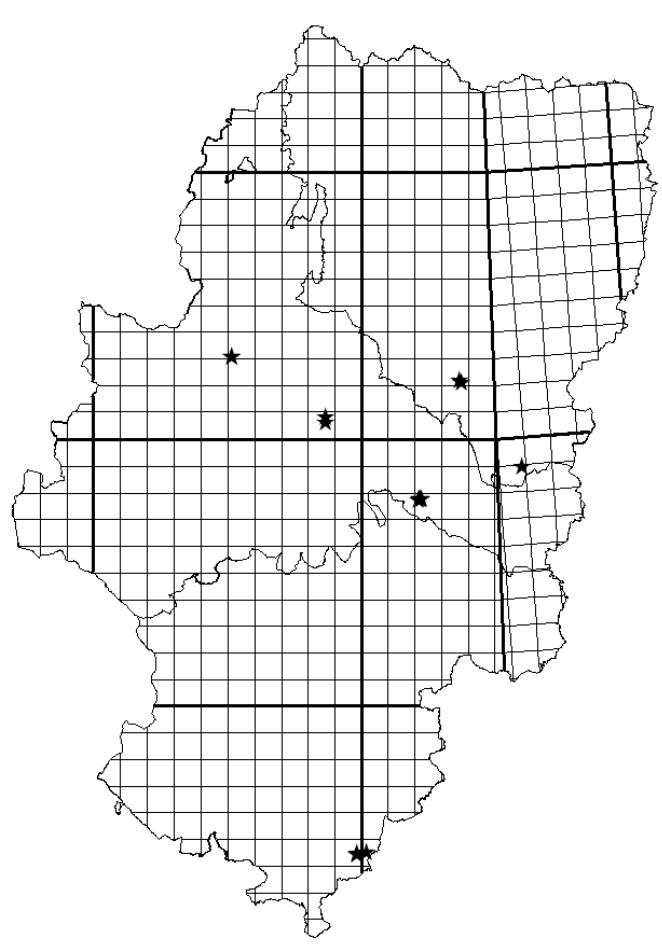

15

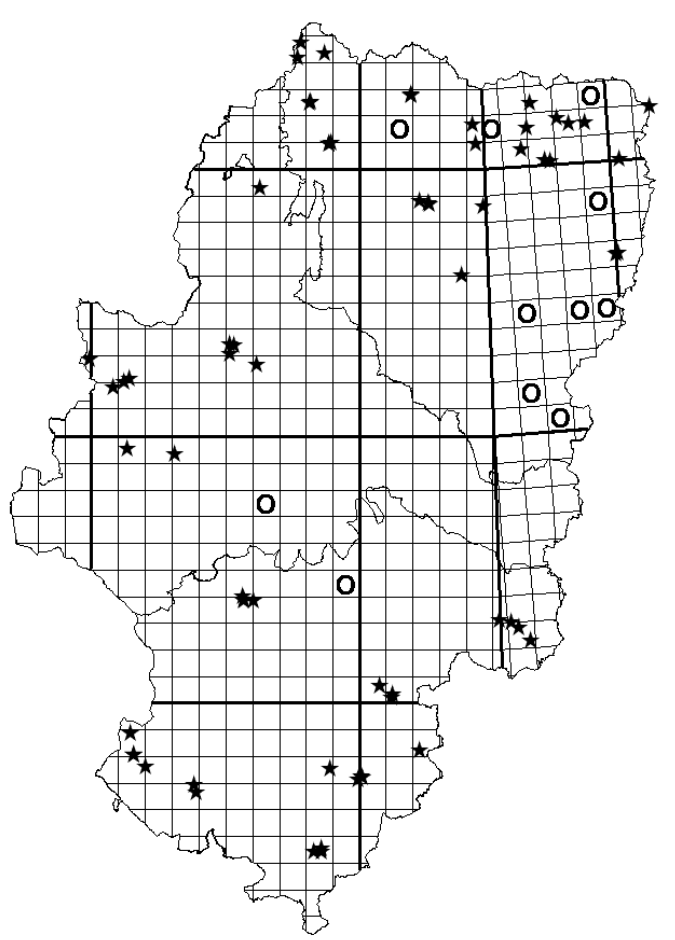

14

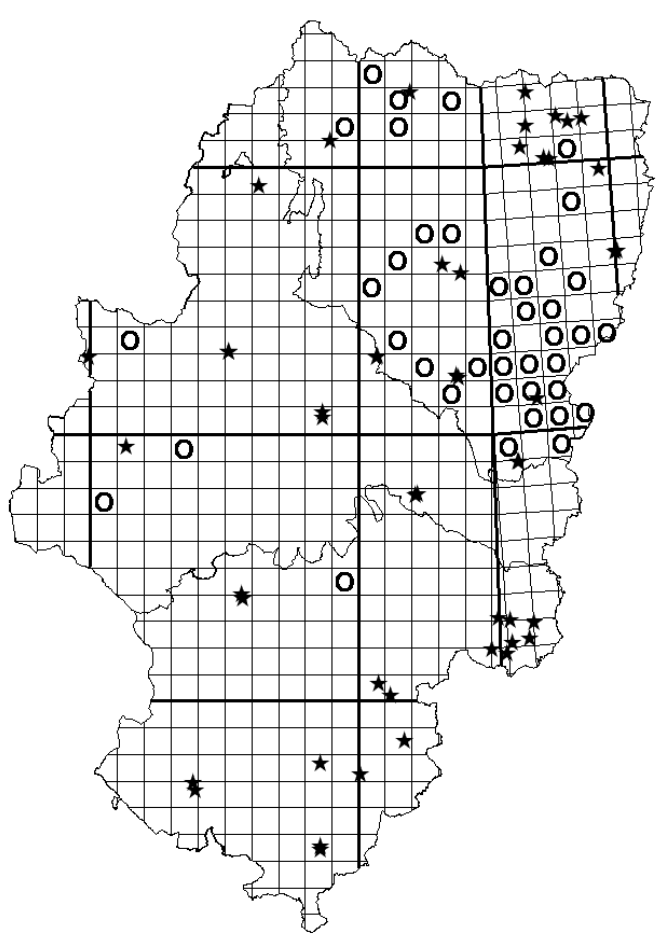

16

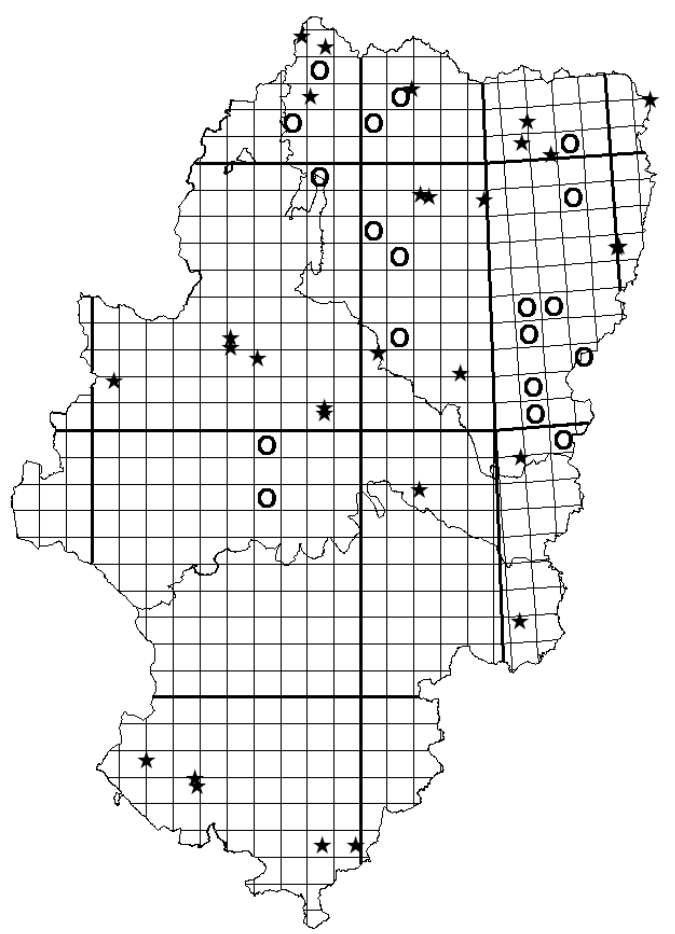

Fig. 13-16.- Observaciones de Pipistrellus pygmaeus (13), P. kuhlii (14), Hypsugo savii (15) y Eptesicus serotinus (16). Símbolos como en la Fig. 2.

Fig. 13-16.- - Records of Pipistrellus pygmaeus (13), P. kuhlii (14), Hypsugo savii (15) y Eptesicus serotinus (16). Symbols as in Fig. 2. 
17

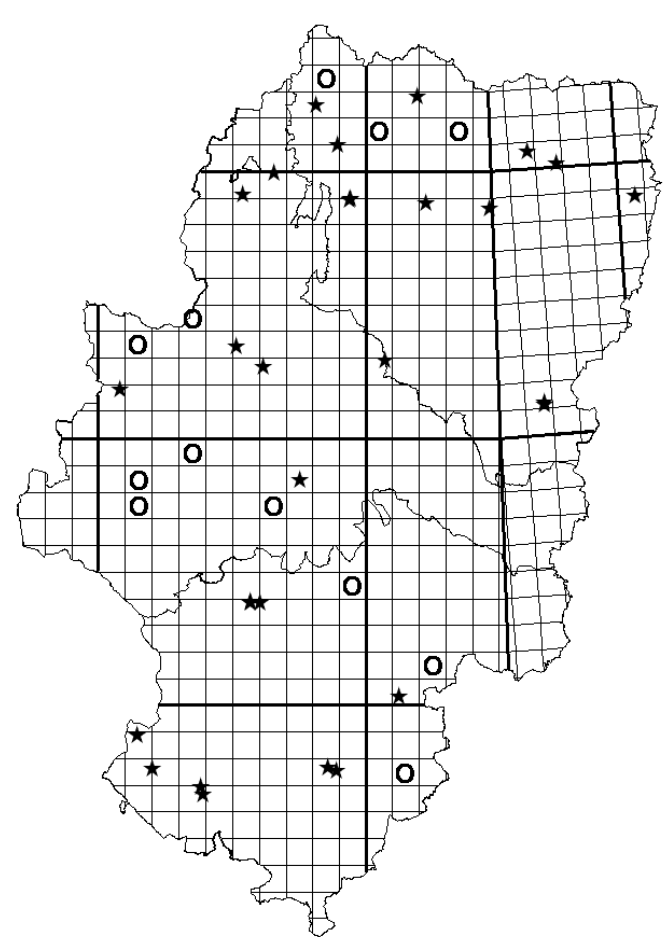

18

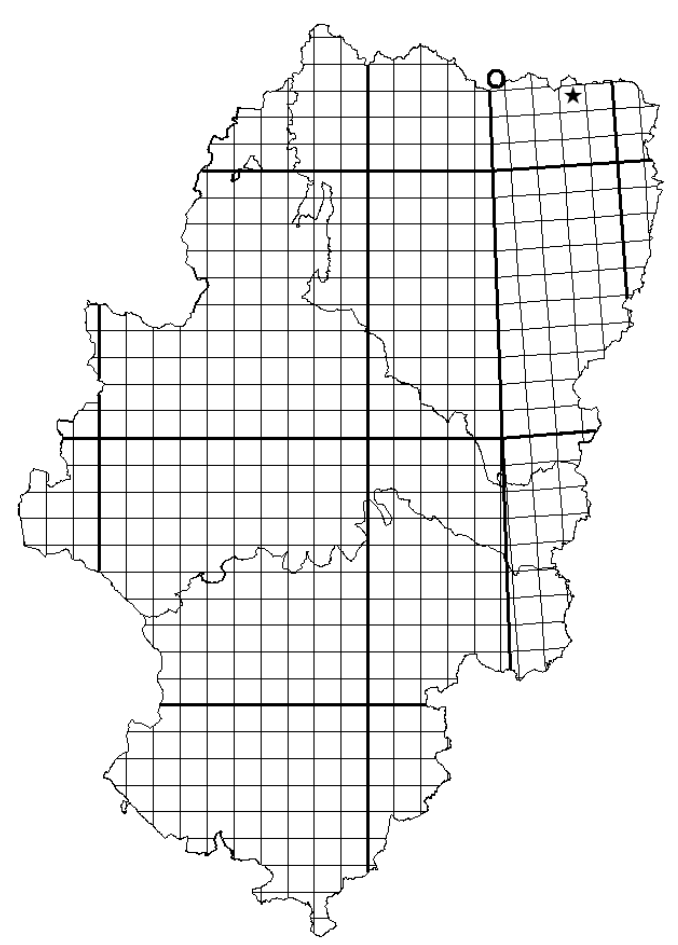

Fig. 17-18.- Observaciones de Plecotus austriacus (17) y P. macrobullaris (18) (estrella: nuevo dato; círculo blanco: dato de Garin et al., 2003): Resto de símbolos como en la Fig. 2.

Fig. 17-18. - Records of Plecotus austriacus (17) and P. macrobullaris (18) (star: new data; open circle: data from Garin et al., 2003). Remaining symbols as in Fig. 2.

nias reproductoras, las abundantes observaciones y su carácter sedentario (Ibáñez, 2002) indican que probablemente también se reproduce en diferentes zonas de la región.

El orejudo gris, Plecotus austriacus (29 citas, 9 colonias, Fig. 17), resulta también frecuente y parece ubiquista, hallándose de forma continua por todo Aragón.

El orejudo alpino, Plecotus macrobullaris, ha sido citado en la península Ibérica recientemente, en Ordesa (Garin et al., 2003). En el presente trabajo se ha identificado en otra localidad elevada del Pirineo (Fig. 18), donde se capturaron 3 hembras (una de ellas, reproductora) y 6 machos. Allí se observaron ejemplares refugiados en las grietas de una borda. Su presencia parece restringida a las zonas altas del Pirineo (por encima de los 1700 m.s.n.m.).

El murciélago rabudo, Tadarida teniotis (40 citas, 1 colonia, Fig. 19), se distribuye por la mayor parte de Aragón. Sus largos desplazamientos y su fácil escucha (emite pulsos de ecolocación audibles y de gran intensidad) favorecen su cita en numerosas localidades; sin embargo, sus efectivos son probablemente más escasos de lo que reflejan sus observaciones.

\section{ESPECIES FORESTALES}

El murciélago ratonero bigotudo, Myotis mystacinus (6 citas, 1 colonia, Fig. 20), es una especie de muy baja detectabilidad, por lo que puede pasar desapercibida en su hábitat. Se ha encontrado en pinares de pino negro y silvestre, hayedos, rebollares y quejigales, generalmente por encima de los $1000 \mathrm{~m}$ de altitud (5 observaciones), en Pirineos y el sur de Teruel.

El nóctulo pequeño, Nyctalus leisleri, se ha observado en 16 localidades del Pirineo y Moncayo, siendo relativamente frecuente en ambos macizos (Fig. 21). Habita principalmente en hayedos, pinares de pino silvestre y robledales $(80 \%$ de las observaciones). Los 49 ejemplares capturados en verano eran machos, por lo que parece que no cría en 
19

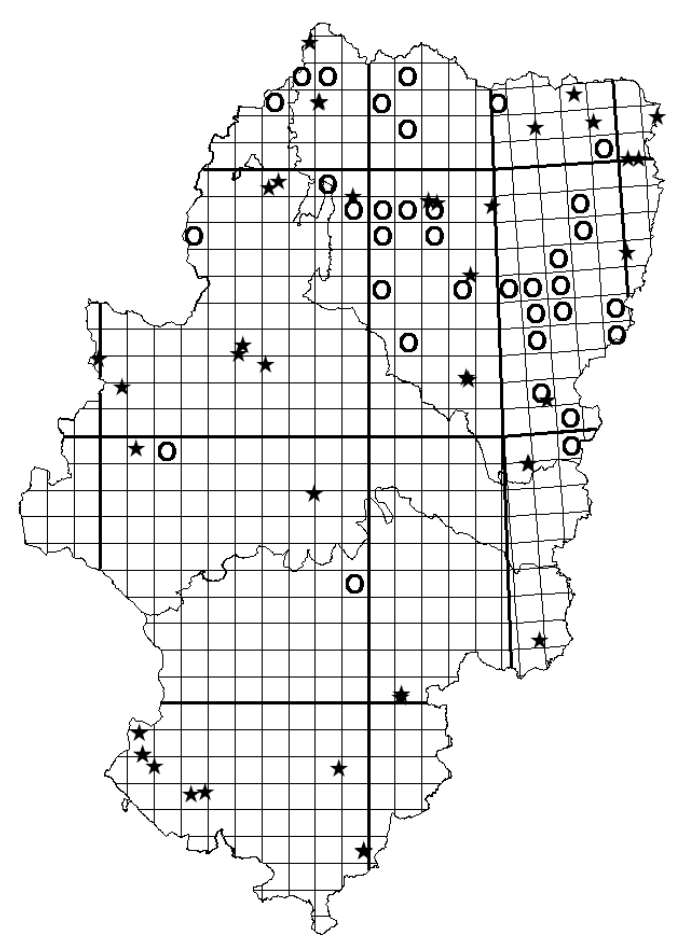

21

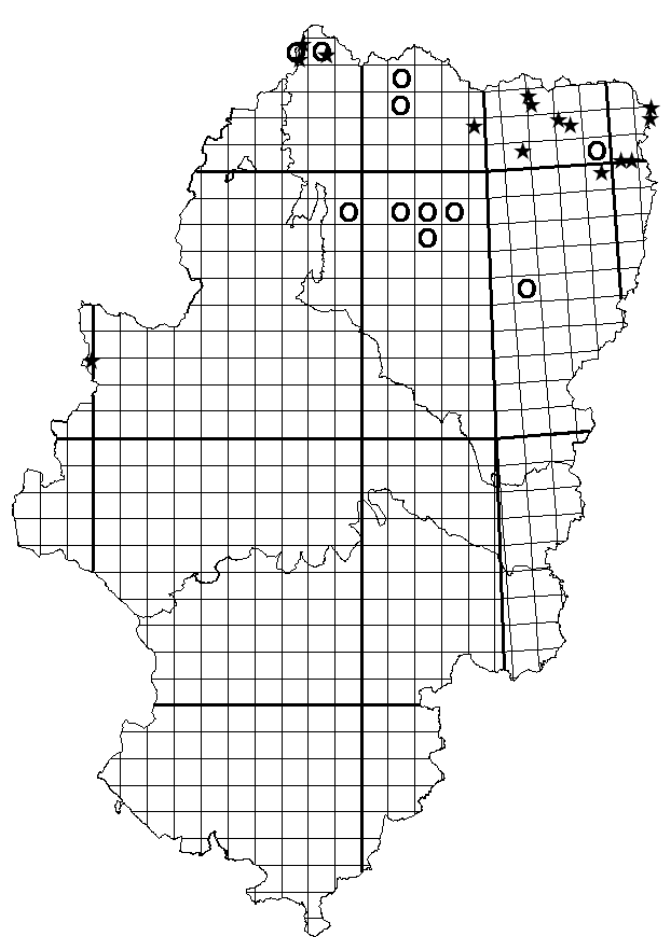

20

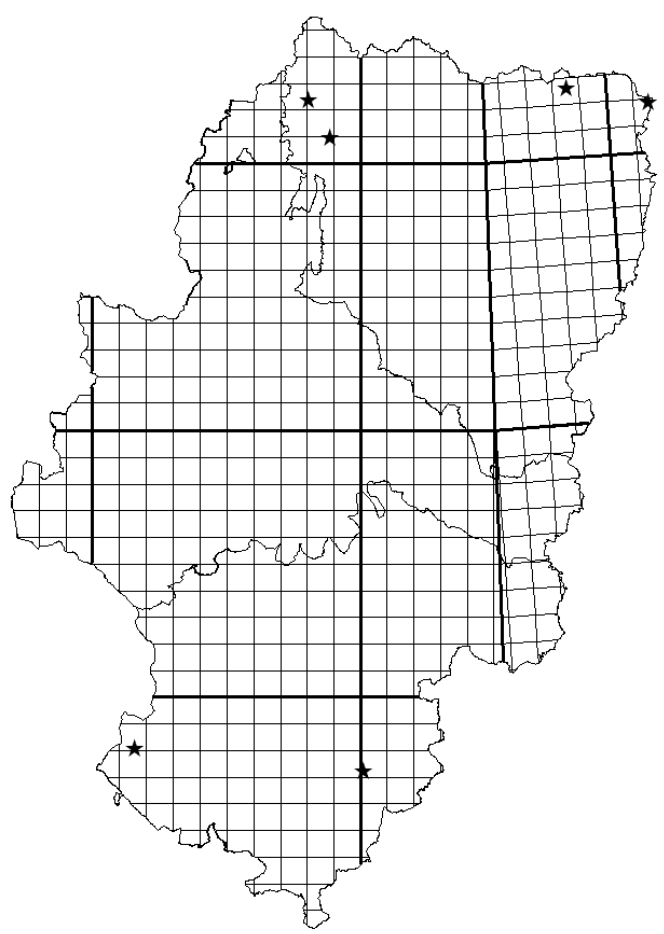

22

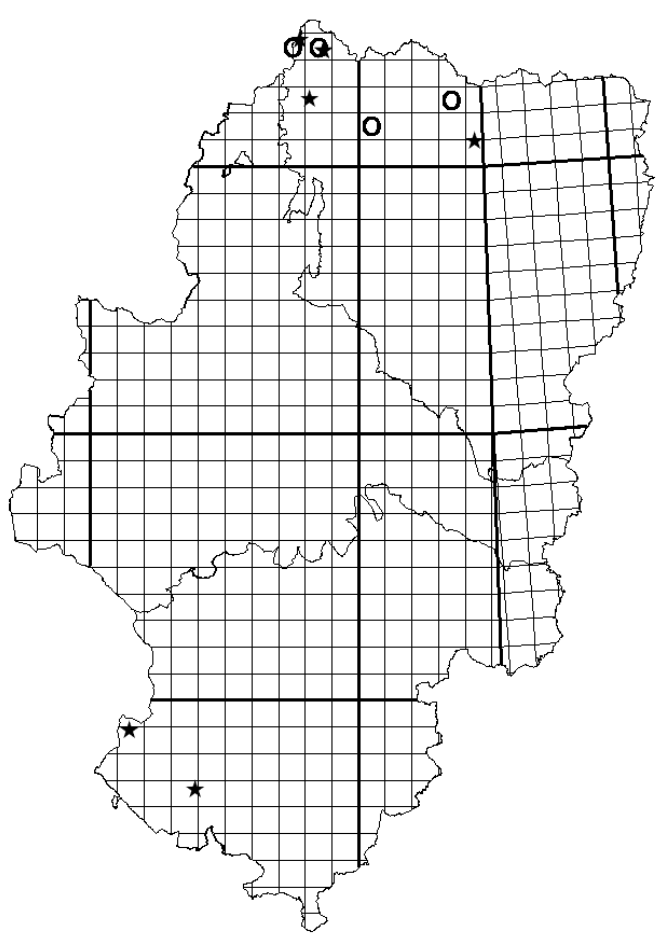

Fig. 19-22.- Observaciones de Tadarida teniotis (19), Myotis mystacinus (20), Nyctalus leisleri (21) y N. lasiopterus (22). Símbolos como en la Fig. 2.

Fig. 19-22.- - Records of Tadarida teniotis (19), Myotis mystacinus (20), Nyctalus leisleri (21) y N. lasiopterus (22). Symbols as in Fig. 2. 
23

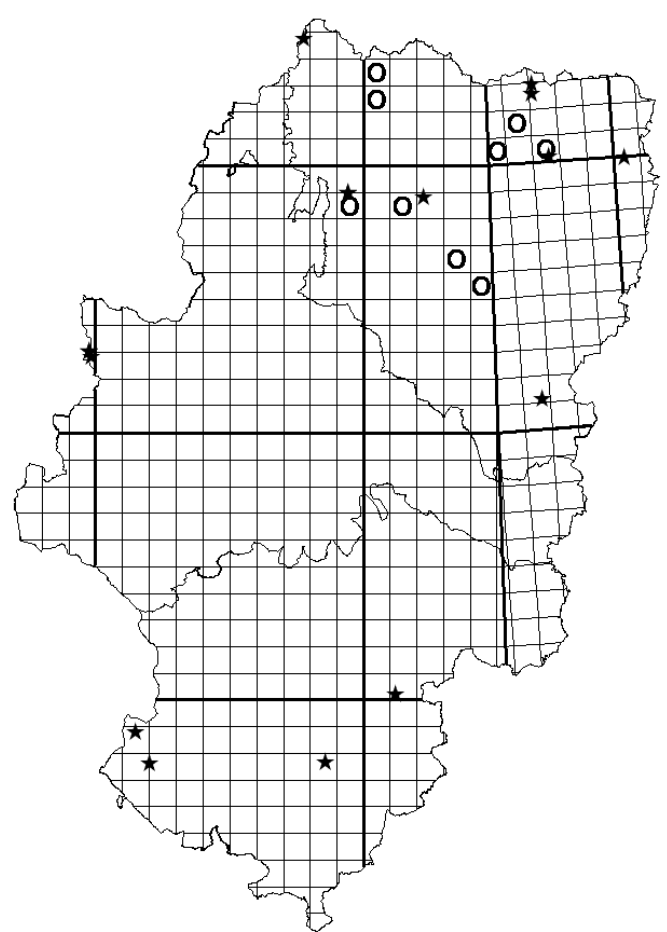

24

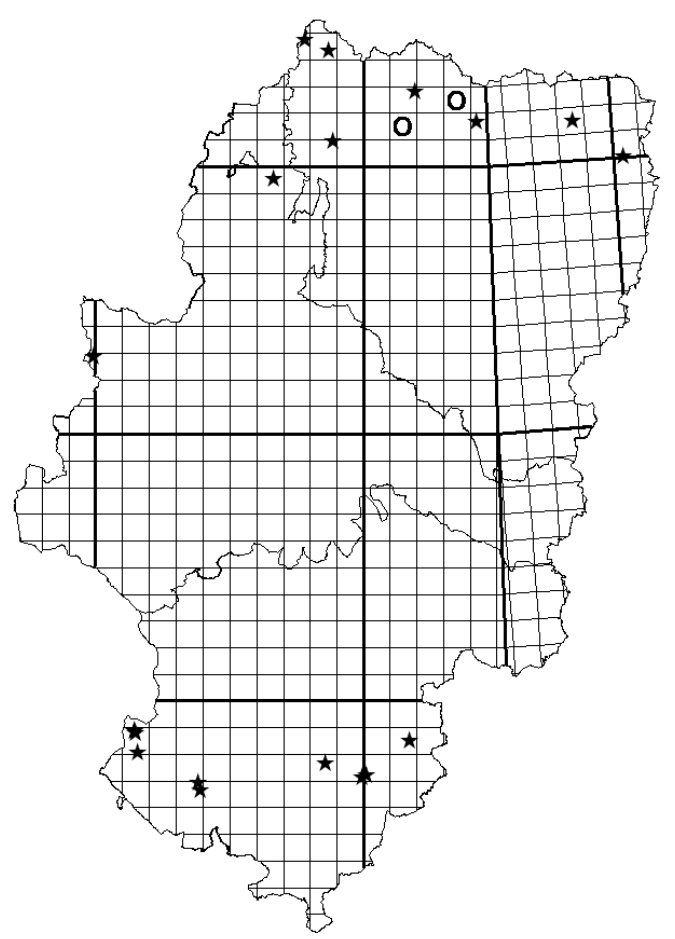

Fig. 23-24.- Observaciones de Barbastella barbastellus (23) y Plecotus auritus (24). Símbolos como en la Fig. 2.

Fig. 23-24. - Records of Barbastella barbastellus (23) and Plecotus auritus (24). Symbols as in Fig. 2.

Aragón, al igual que ocurre en otras comunidades vecinas como Navarra (Alcalde, 1995), La Rioja (Agirre-Mendi, 2002) y País Vasco (Aihartza, 2001).

El nóctulo grande, Nyctalus lasiopterus (7 citas, Fig. 22), se ha capturado en bosques diversos (rebollares, pinares de pino silvestre y de pino piñonero, hayedos) de Pirineos y Albarracín. Todas las capturas se han realizado en verano y todos los ejemplares (16) eran machos. Aunque existen evidencias de su cría (hembras lactantes) en el Parque Nacional de Ordesa (Aihartza, com. pers., 2004), probablemente, el número de colonias reproductoras debe ser muy reducido.

El barbastela, Barbastella barbastellus, y el orejudo dorado, Plecotus auritus, habitan en las tres áreas más boscosas de Aragón (Pirineos, Moncayo y sierras del sur de Teruel) donde parecen relativamente comunes. El barbastela (16 citas y 4 colonias identificadas, Fig. 23) predomina en pinares de pino silvestre, hayedos y robledales $(80 \%$ de las observaciones) aunque también se halla en bosques de baja altitud como algunos sotos maduros del río Alcanadre. La única colonia localizada se encontraba en las contraventanas de un edificio abandona- do, en un bosque mixto de hayas, pinos silvestres y robles, y contaba con 30 ejemplares aproximadamente. Plecotus auritus está restringida a zonas situadas por encima de los $1000 \mathrm{~m}$ de altitud (tabla 2), donde puede ser frecuente (19 citas y 9 colonias identificadas, Fig. 24) principalmente en pinares de pino silvestre y hayedos (63\% de las localidades). Sus dos subespecies se reparten alopátricamente: $P$. $a$. auritus se encuentra únicamente en Pirineos, mientras que $P$. a. begognae se halla en el centro (Moncayo) y sur de Aragón.

\section{Discusión}

Los datos obtenidos, junto con los procedentes de la bibliografía, permiten agrupar la mayoría de las especies en cuatro patrones básicos de distribución, así como deducir el estado de conservación de sus poblaciones:

- Especies frecuentes, de distribución general y continua por toda la región: Pipistrellus pipstrellus, Pipistrellus kuhlii, Hypsugo savii, Eptesicus serotinus, Plecotus austriacus y Tadarida 
teniotis. Todas son fisurícolas y sus poblaciones no están amenazadas.

- Especies menos frecuentes, pero de distribución general y dispersa por Aragón: Rhinolophus ferrumequinum, Rhinolophus hipposideros, Rhinolophus euryale, Myotis myotis, Myotis blythii, Myotis escalerae, Myotis emarginatus y Miniopterus schreibersii. Todas son cavernícolas y por lo general, sus poblaciones se agrupan en unas pocas colonias, por lo que resultan vulnerables.

- Un grupo de especies forestales se hallan restringidas a los grandes macizos montañosos y boscosos: Myotis mystacinus, Nyctalus leisleri, Nyctalus lasiopterus, Barbastella barbastellus y Plecotus auritus. Las dos últimas no poseen poblaciones muy numerosas, pero sus colonias se dispersan por diferentes bosques, por lo que no parecen amenazadas. De las demás especies, se tienen muy pocos datos y su situación es incierta: $N$. leisleri es frecuente pero parece no criar en Aragón. De M. mystacinus sólo consta una colonia reproductora, al igual que de $N$. lasiopterus (Aihartza, com. pers., 2004), y sus poblaciones podrían estar amenazadas.

- Especies de distribución reducida: dos de ellas son muy frecuentes en las zonas que habitan y por ello no se consideran amenazadas (Myotis daubentonii y Pipistrellus pygmaeus), mientras que otras tres son todavía poco conocidas y no se puede determinar su estado (Myotis cf. nattereri, Myotis capaccinii y Plecotus macrobullaris).

Igualmente, se desconoce la situación de otras especies: Pipistrellus nathusii y Nyctalus noctula han sido citadas una sola vez, con un único ejemplar capturado en Huesca (Woutersen \& Bafaluy Zoriguel, 2001). Dichos autores también citan a Myotis bechsteinii en la misma provincia, aunque sin aportar datos biométricos o documento fotográfico en el que claramente pueda identificarse la especie; no obstante, Alcocer \& Monsalve (com. pers., 2006) registraron y fotografiaron algunos ejemplares en tres localidades del sur de Teruel, lo que certifica su presencia en la región. La escasez de datos a pesar de los intensos muestreos, hace sospechar que sus poblaciones sean muy reducidas.

En resumen, las especies más frecuentes y abundantes son las fisurícolas y especialmente las adaptadas a entornos humanos, repartiéndose generalmente por toda la región de forma continua. Las especies cavernícolas son más escasas, se distribuyen de forma discontinua y sus poblaciones se con- centran entorno a las zonas con abundancia de cuevas, minas y construcciones apropiadas. Por último, los quirópteros forestales parecen ser los menos frecuentes y por lo general están presentes únicamente en las áreas boscosas de mayor extensión y donde además existen numerosos árboles de tamaño y edad considerable (Pirineos, Moncayo y sierras del sur de Teruel); por el contrario, faltan en el valle del Ebro, donde el terreno está muy humanizado, dedicado principalmente a cultivos intensivos, y la mayoría de los bosques son pinares o encinares con ejemplares poco desarrollados.

\section{AGRADECIMIENTOS}

El presente estudio ha sido financiado por el Departamento de Medio Ambiente del Gobierno de Aragón. Queremos agradecer además la colaboración de numerosas personas que nos han facilitado información y nos han ayudado en la localización de los puntos de muestreo: Paloma Barrachina, Joaquín Guerrero, Manuel Mercadal, Marirrós Gallego, Javier Gascón, Miguel Ortiz, Félix Compaired, José Urbano, José Antonio Bardají, Miguel Arregui, Ignacio García, Ignacio Marín, Alberto Portero, Pedro Vicente Ruiz Sánchez, Francisco Sebastián, José Luis Aznar, Vicente Martín, Luis Iriarte, Antonio Fau, Jesús Gracia, Jerónimo Navarro, Óscar Lacosta y Jorge Abanto.

Queremos agradecer especialmente a los investigadores de la Estación Biológica de Doñana, Carlos Ibáñez, Javier Juste, Juan L. García-Mudarra, Irene Salicini y María Napal, la realización de análisis genéticos que han permitido determinar con seguridad algunas especies crípticas.

Por último agradecemos a Teresa Gil su hospitalidad y simpatía y a Félix González, José Antonio Garrido y Óscar de Paz por facilitarnos el acceso a los datos del Atlas de los Mamíferos terrestres de España.

\section{Referencias}

AgirRe-Mendi, P. T., 2002. Nyctalus leisleri (Kuhl, 1817). In: L. Palomo \& J. Gisbert (eds.). Atlas de los Mamíferos terrestres de España. DGCN-SECEMSECEMU. Madrid: 194-197.

AgIRRE-Mendi, P. T. \& IBÁÑEZ, C., 2004. Distribución de Pipistrellus pipistrellus (Schreber, 1775) y Pipistrellus pygmaeus (Leach, 1925) en la Comunidad Autónoma de La Rioja. Zubía, 22: 101-111.

AHLÉN, I., 1990. Identification of bats in flight. Swedish Society for Conservation of Nature and The Swedish Youth Association for Environmental Studies and Conservation. Katarina Tryck AB. Estocolmo. 50 pp.

Ainartza, J. R., 2001. Quirópteros de Araba, Bizkaia y Gipuzkoa: Distribución, Ecología y Conservación. Tesis Doctoral. Universidad de Leioa. 346 pp.

Alcalde, J. T., 1995. Distribución y fenología de los quirópteros de Navarra. Tesis Doctoral. Universidad de Navarra. 430 pp. 
Almenar, D., Alcocer, A. \& Monsalve, M. A., 2002. Myotis capaccinii (Bonaparte 1837). In: L. Palomo \& J. Gisbert (eds.). Atlas de los Mamíferos terrestres de España. DGCN-SECEM-SECEMU. Madrid: 170-173.

BAFAluy Zoriguel, J. J., 1997. Informe sobre la importancia del Canal de Aragón y Cataluña para los quirópteros y medidas recomendadas para la conservación de los refugios. ANSAR. Monzón. Informe inédito.

BAfAluy Zoriguel, J. J., 1999. Los murciélagos en el área del Bajo Cinca. (Huesca, Zaragoza, Lleida). CINGA, Anuari de l'Institut d'Estudis del Baix Cinca, 3: 83-100.

BAfAluy Zoriguel, J.J., 2000. Mortandad de murciélagos por atropello en carreteras del sur de la provincia de Huesca. Galemys, 12: 15-23.

Bafaluy Zoriguel, J. J., LaVedÁn Rodríguez, J. \& Moreno Rodríguez, J. D., 1997. Murciélagos de la cuenca media del Cinca. (Primera aproximación a su estudio). CEHIMO, 24: 255-271.

BAlcells, E., 1965. Nuevos datos sobre murciélagos raros en cuevas españolas. Miscellánea Zoológica, 2: 149-160.

Barataud, M., 1996. The World of Bats. Sittelle Press. Mens. 47 pp.

Boyero, J. R., 2002. Myotis daubentonii (Kuhl, 1817). In: L. Palomo \& J. Gisbert (eds.). Atlas de los Mamíferos terrestres de España. DGCN-SECEMSECEMU. Madrid: 166-169.

CABrera, A., 1914. Fauna Ibérica: Mamíferos. Museo Nacional de Ciencias Naturales. Madrid. 441 pp.

Garin, I., García-Mudarra, J. L., Aihartza, J. R., Goiti, U. \& Juste, J., 2003. Presence of Plecotus macrobullaris (Chiroptera: Vespertilionidae) in the Pyrenees. Acta Chiropterologica, 5(2): 243-250.

Goiti, U. \& Garin, I., 2002. Pipistrellus kuhlii (Kuhl, 1817). In: L. Palomo \& J. Gisbert (eds.). Atlas de los Mamíferos terrestres de España. DGCN-SECEMSECEMU. Madrid: 182-185.

IBÁÑEZ, C. 2002. Eptesicus serotinus Schreber, 1774. In: L. Palomo \& J. Gisbert (eds.). Atlas de los Mamíferos terrestres de España. DGCN-SECEM-SECEMU. Madrid: 206-209.

IBÁÑEZ, C. \& FERNÁNDEZ, R., 1989. Catálogo de murciélagos de las colecciones del Museo Nacional de Ciencias Naturales. Museo Nacional de Ciencias Naturales, CSIC. Madrid. 54 pp.

IbÁÑEz, C., GarcíA-MudarRa, J. L., Ruedi, M., StAdelMAN, B. \& JUSTE, J., 2006. The iberian contribution to cryptic diversity in European bats. Acta Chiropterologica, 8(2): 277-297.
Kunz, T. H. \& KurTA A., 1988. Capture methods and holding devices. In: T. H. Kunz (ed.). Ecological and behavioral methods for the study of bats. Smithsonian Institution Press. Washington: 1-29.

MAYNAR, J., 1919. Nota sobre algunos animales hallados en la cueva de los Encantados (Zaragoza). Boletín de la Real Sociedad Española de Historia Natural, 19: 164.

NATURe Conservancy Council, 1987. The bat worker's manual. A. J. Mitchell-Jones. Peterborough. 108 pp.

NAVAS, L., 1901. La cueva de Maderuela en Vera (provincia de Zaragoza). Continuación. Boletín de la Real Sociedad Española de Historia Natural, 1: 25-31.

Palomo, L. J. \& Gisbert, J. (eds.), 2002. Atlas de los Mamíferos terrestres de España. Dirección General de Conservación de la Naturaleza-SECEM-SECEMU. Madrid. 564 pp.

Russo, D. \& Jones, G., 2002. Identification of twentytwo bat species (Mammalia: Chiroptera) from Italy by analysis of time-expanded recordings of echolocation calls. Journal of Zoology, London, 258: 91103.

Serra-Cobo, J., Amengual-Pieras, B. \& EstradAPEÑA, A., 1992. Nuevos datos sobre los quirópteros de Aragón. In: A. Alemany (ed.). Historia Natural'91. Universitat de les Illes Balears. Palma: 229-236.

TrujILlo, D., 1991. Murciélagos de las Islas Canarias. ICONA, Colección Técnica. Madrid. 167 pp.

Woutersen, K. \& Bafaluy Zoriguel, J. J., 2001. Murciélagos del Alto Aragón. Kees Woutersen Publicaciones. Huesca. 144 pp.
Recibido, 3-V-2007 Aceptado, 31-I-2008 Publicado, 30-VI-2008 